\title{
Unterschiedliche Rahmenbedingungen bei der Realisierung von Maturaarbeiten
}

\section{Christina Huber, Lukas Lehmann und Vera Husfeldt}

Das Maturitätsanerkennungsreglement (MAR) von 1995, ein gemeinsam konzipiertes Regelwerk von Bund und Kantonen, wurde 2007 teilreformiert. Die Teilreform betraf unter anderem die mit dem MAR 1995 eingeführte Maturaarbeit: War sie bis anhin als der eigentlichen Maturaprüfung vorangehende Projektarbeit in die Ausbildung eingeflossen, zählt sie neu auch als Abschlussnote der gymnasialen $M a-$ turität. Damit muss die Frage nach der Vergleichbarkeit von Maturaarbeiten und ibren Bewertungen gestellt werden.

Im Rahmen der Evaluation der Maturitätsreform (EVAMAR II) wurde die Maturitätsbildung bereits zum zweiten Mal gesamtschweizerisch evaluiert. Dieses Mal wurden auch die Maturaarbeiten einer vertieften Überprüfung unterzogen. Der folgende Artikel stellt ausgewählte Ergebnisse aus dieser Studie vor.

\section{Die Maturaarbeit im gymnasialen Kontext}

Bereits 1969 schlug eine EDK-Studienkommission im Bericht «Mittelschule von Morgen» (EDK, 1972) die Einführung schriftlicher Arbeiten zur individuellen Vertiefung und zur verbesserten Vorbereitung auf akademische Bildungsgänge vor. Rechtlich verbindend eingeführt wurden solche Maturaarbeiten aber erst im Zuge der Revision der Maturitätsanerkennungsverordnung in den 1990er Jahren (MAR 95, Art. 10).

Die Maturaarbeit leistet einen Beitrag zum dreifachen gymnasialen Bildungsauftrag, der eine vertiefte Allgemeinbildung, die Ausbildung einer allgemeinen Studierfähigkeit bzw. Hochschulreife sowie die wissenschaftspropädeutische Bildung umfasst (vgl. KMK, 2006; MAR 95, Art. 5). Was sich hinter diesen Konstrukten genau verbirgt, bedarf der Klärung. Wolter (1997, S. 49) stellt die These auf, dass sich hinter dem Konstrukt der Studierfähigkeit ein unauflösliches und mehrdimensionales Heterogenitätsproblem verbirgt, das seine Ursache einerseits in den Ausdifferenzierungs- und Spezialisierungsprozessen des Wissenschaftssystems und andererseits in gesamtgesellschaftlichen Entwicklungen (Plu- 
ralisierung der Informations- und Kommunikationswege sowie der alltäglichen Lebenswelten und Sozialisationsfelder) hat. Sowohl in Bezug auf die Bildungsvoraussetzungen und Erwartungen der Studierenden als auch bei den Studienanforderungen und den Erwartungen der Hochschulen stellt er eine Zunahme an Heterogenität fest. Empirische Untersuchungen verweisen darauf, dass die einzelnen Disziplinen tatsächlich unterschiedliche Erwartungen an ihre Studienanfängerinnen und -anfänger haben (Heldmann, 1998; Konegen-Grenier, 2002). Aufgrund einer Befragung von Hochschuldozierenden kommt KonegenGrenier (2002, S. 168) dennoch zu einer allgemeinen inhaltlichen Bestimmung von Studierfähigkeit, welche die formalen Dimensionen , analytische Fähigkeiten', ,Abstraktionsfähigkeit' und ,Differenzierungsvermögen' umfasst. Inhaltlich werden gute Kenntnisse in den Fächern Englisch, Mathematik und Deutsch ebenso als wichtig erachtet wie die Kenntnis grundlegender Arbeitstechniken (Präsentationsfähigkeit, Kenntnisse in Textverarbeitung und Recherchetechniken). Dieser Versuch einer Bestimmung des Konstrukts der Studierfähigkeit deutet die enge Verknüpfung, aber auch die fehlende Trennschärfe in Bezug auf das Konstrukt der Wissenschaftspropädeutik an. Häufig wird Wissenschaftspropädeutik auf der Basis methodologischer Aspekte beschrieben (so etwa die Operationalisierung im Rahmen der TOSCA-Studie, Trautwein \& Lüdtke, 2004; oder das Konzept der «Scientific Literacy», National Academy of Science, 1996). Dies verweist darauf, dass Wissenschaftspropädeutik vor allem zu Methodenbewusstsein und -bewusstheit führen soll (Huber, 1997; Schmidt, 1994).

Die Maturaarbeit dient der Aneignung solcher Kompetenzen, zumal es beim Prozess des Erstellens einer Maturaarbeit darum geht, individuell oder im Team über einen längeren Zeitraum projektbezogen zu arbeiten, sich vertieft mit ausgewählten Methoden des Recherchierens, Analysierens und Argumentierens vertraut zu machen und nachzuweisen, dass man elementare wissenschaftliche Informationen sachgerecht verarbeiten kann. Die Erwartungen an die Maturaarbeiten erstrecken sich dementsprechend nicht nur auf rezeptive und wissensverarbeitende Leistungen, sondern auch auf die Performanz: Das im Prozess erworbene Wissen muss angemessen kommuniziert werden, d.h. die Maturarbeit ist gleichzeitig auch als Leistungsnachweis zu werten, welcher den erfolgreichen Erwerb dieser Kompetenzen dokumentiert. Damit nimmt sie eine Doppelfunktion als Element des Kompetenzaufbaus und -nachweises ein.

\section{Der «neue» Stellenwert der Maturaarbeit}

Die Maturaarbeit hatte bis vor kurzem für das Erlangen des Maturitätszeugnisses nur eine Nebenrolle gespielt. Mit der im Juli 2007 beschlossenen Teilrevision des MAR (EDK, 2007) zählt sie aber seit dem Schuljahr 2008/09 zur Abschlussnote der gymnasialen Maturität. Mit der Aufwertung wurde dem Wunsch einer Mehrheit von Gymnasiastinnen und Gymnasiasten (Binder \& Feller-Länz- 
linger, 2003, 2004; Davaud \& Hexel, 2003; Pagnossin, Alliata \& Dozio, 2005; USO, 2006) sowie einer Forderung des Vereins Schweizerischer Gymnasiallehrerinnen und Gymnasiallehrer (VSG) entsprochen (Dreyer, 2007). Dennoch: Eine noch vor der Reform durchgeführte Befragung von Aargauer Gymnasiallehrpersonen zeigte, dass diese den Verzicht auf die prüfungsrelevante Anrechung mehrheitlich eher bevorzugt hätten, auch wenn sie dem Anliegen der Schülerinnen und Schüler grundsätzlich folgen konnten. Nebst der grundsätzlichen Kritik gegen eine Dominanz von Noten wurde damit auch darauf aufmerksam gemacht, dass die Höhergewichtung der Maturaarbeit eine Bewertung erfordert, die verbindlichen und vergleichbaren Standards zu entsprechen vermag (Binder \& Feller-Länzlinger, 2004). Während nämlich für «konventionelle» Maturafächer formale sowie inhaltliche Vorgaben im Zielartikel des MAR und im Rahmenlehrplan (EDK, 1994) - wenn auch in nur wenig operationalisierter und lose verbindlicher Form (Eberle et al., 2008) - umschrieben und in den kantonalen Lehrplänen verankert sind, fehlen ebensolche schulübergreifenden Zielbestimmungen und Verfahrensrichtlinien für die Maturaarbeit ganz. Dass bezüglich Zielsetzungen und Verfahrensgrundsätzen wenig Einigkeit besteht, zeigt auch die im Rahmen von EVAMAR II durchgeführte Untersuchung zur Qualität von Maturaarbeiten (Huber, Husfeldt, Lehmann \& Quesel, 2008).

\section{EVAMAR II-Teilprojekt D2: Qualität von Matura- arbeiten}

Mit der Untersuchung der Qualität von Maturaarbeiten (Huber et al., 2008) sollten Forschungslücken geschlossen werden, indem die Maturaarbeiten selbst im Fokus der Untersuchung standen. Ausgangspunkt der Untersuchung war ein Wirkungsmodell, das den «Produktionsprozess» der Maturaarbeiten erfasst (vgl. Abb. 1).

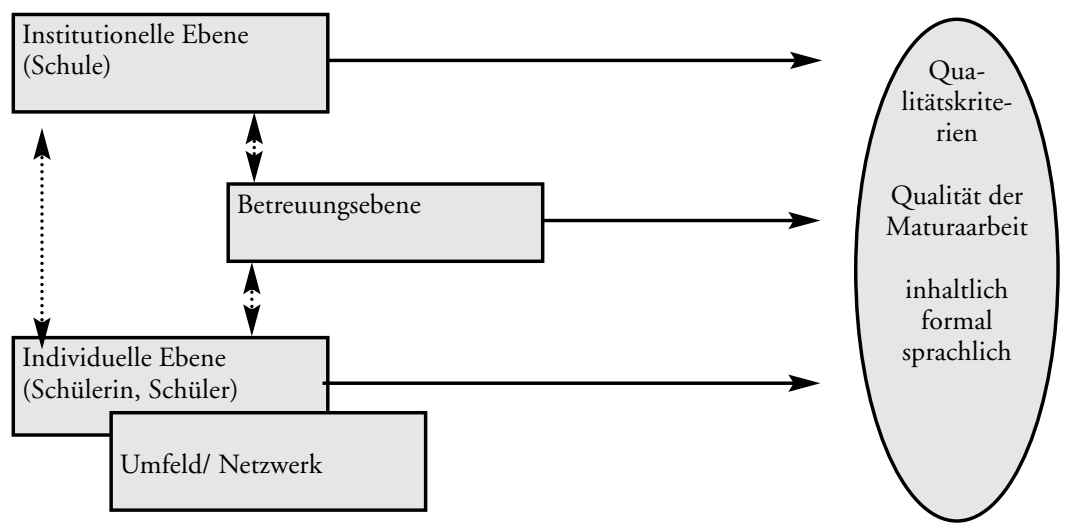

Abbildung 1: Wirkungsmodell zur Genese der Maturaarbeiten 
Als relevante Faktoren im Hinblick auf die Qualität der Arbeiten wurden die institutionellen Rahmenbedingungen, die Betreuung während des Arbeitsprozesses sowie die Schülerinnen und Schüler als eigentliche Produzenten der Arbeit ausgemacht.

\section{Untersuchungsdesign}

Auf der Grundlage des Wirkungsmodells (Abb. 1) wurde die Untersuchung in drei Teilkomponenten gegliedert. Im Zentrum stand die Analyse der Qualität von Maturaarbeiten anhand eines Kriterienrasters (vgl. Abschnitt 3.3).

Flankierend wurden zudem die institutionellen Rahmenbedingungen in einer komparativen Perspektive untersucht. Dabei wurden nationale, kantonale sowie einzelschulische Vorgaben unterschieden. Auf nationaler Ebene wurden das damals geltende Maturitätsanerkennungsreglement (EDK, 1995) sowie der Rahmenlehrplan für die Maturitätsschulen (EDK, 1994) und auf kantonaler Ebene die entsprechenden Gesetze und Verordnungen analysiert. Zudem wurden auch die kantonalen Lehrpläne im Hinblick auf die Thematik untersucht und eine Inhaltsanalyse der durch die Schulen schriftlich dokumentierten Informationen (Weisungen, Reglemente, Leitfäden usw.) vorgenommen.

Des Weiteren wurden Maturandinnen und Maturanden mittels eines standardisierten Fragebogens schriftlich befragt. Dabei interessierten neben soziodemographischen Daten und allgemeinen Angaben zur Maturaarbeit (Thema und Themenwahl, Bewertung der Arbeit durch Lehrperson) vor allem die Betreuungssituation (Form und Häufigkeit der Betreuung, Zufriedenheit mit der Betreuung) und Aspekte der Unterstützung und Ressourcen (Zufriedenheit mit den schulischen Rahmenbedingungen, Unterstützungsangebote, soziales Netzwerk). Ausserdem wurden die Schülerinnen und Schüler um eine Einschätzung ihres individuellen Aufwands und Nutzens der Maturaarbeit gebeten.

\section{St ichprobe}

Die Grundgesamtheit der Untersuchung setzt sich aus allen Gymnasiastinnen und Gymnasiasten zusammen, die im Schuljahr 2006/07 ihre Maturaarbeit verfasst haben. Die Stichproben basieren nicht auf einer Zufallsauswahl, sondern auf einer systematischen Ziehung: Die Ziehung erfolgte in einem ersten Schritt auf der Ebene der Schulen. Um sicherzustellen, dass alle drei Sprachregionen angemessen vertreten sind, wurde zudem eine Schichtung nach Sprachregionen vorgenommen. Innerhalb dieser expliziten Strata wurden die Schulen so sortiert, dass Schulen aus gleichen Kantonen benachbart sind (implizite Stratenbildung). Die Ziehung erfolgte dann ausgehend von einer Zufallszahl. Darauf folgend wurden pro Schule mittels Zufallsverfahren zwei Klassen gezogen.

Aufgrund der Tatsache, dass rund drei Viertel aller Maturaarbeiten theoretischer oder empirischer Natur sind (Pagnossin et al., 2005) und aufgrund der Überlegung, dass ein Einbezug von soziokulturellen Projekten sowie künstlerischen, audiovisuellen und materiellen Produktionen zu Problemen der Vergleichbarkeit zwischen den Arbeiten führen würde, wurde eine Einschränkung 
der Ziehung auf theoretische und empirische Maturaarbeiten versucht. Diese Kategorisierung wurde von den untersuchten Schulen selbst durchgenommen, was sich in der Praxis der Durchführung letztlich als problematisch heraus stellte, da eine trennscharfe Kategorisierung nicht möglich war. So gelangten in unsere Stichprobe auch Arbeiten, die Berichte zu soziokulturellen Projekten, künstlerischen Produktionen und dergleichen umfassten, weil sie von den untersuchten Schulen als empirische Arbeiten klassifiziert wurden. Für die Schülerinnen- und Schülerbefragung wurden die ausgewählten Klassen komplett in die Untersuchung miteinbezogen.

Das angewendete Stichprobendesign gibt aus pragmatischen Gründen vor, dass aus jeder der gezogenen Schulen zwei ganze Klassen untersucht werden. Es handelt sich insofern um eine geklumpte Stichprobe. Da Fälle in einer Klumpenstichprobe sich mehr ähneln als in einer reinen Zufallsstichprobe sollten in ersterer die Fallzahlen höher sein, um trotz Designeffekt eine repräsentative Heterogenität erfassen zu können. Zur Planung der Stichprobengrösse wurde im Vorfeld festgelegt, dass der Mittelwert der Stichprobe im Bereich von $\pm 0,2$ Standardabweichung (SD) des Mittelwertes der Grundgesamtheit liegen sollte, um noch mit einiger Genauigkeit von der Stichprobe auf die Grundgesamtheit schliessen zu können. Um eine ausreichende Genauigkeit (95\%) von Aussagen über Mittelwerte zu erhalten, musste also eine Stichprobengrösse von ca. 100 zugrunde gelegt werden. Unter Berücksichtigung der Klumpenstruktur muss damit gerechnet werden, dass die effektive Stichprobengrösse geringer ausfällt als die reine Anzahl der Fälle. Der Stichprobenplan sah deshalb 450 Fälle vor um auch noch Designeffekte von bis zu deff=4,5 ausgleichen zu können.

Da wir Fallzahlen erreichen wollten, welche die Untersuchung von Relationen zwischen der Wahrnehmung der Schülerinnen und Schüler und den schulischen Rahmenbedingungen erlauben, wurde zudem auch in Kauf genommen, dass Schülerinnen und Schüler aus kleineren Schulen in der Stichprobe überproportional vertreten sind. Dies erscheint legitim, zumal davon ausgegangen werden kann, dass der Faktor Schulgrösse keinen Einfluss auf die Qualität von Maturaarbeiten hat. Für Berechnungen, welche andere, unseres Erachtens relevante Faktoren wie etwa das Geschlecht oder die Sprachregion, berücksichtigen haben wir die Teilstichproben entsprechend gewichtet (zur ausführlichen Beschreibung der Stichprobe siehe Huber et al., 2008, S. 301ff).

Tabelle 1: Grundgesamtheit und Stichprobe der Untersuchung

\begin{tabular}{|l|r|r|r|l|l|}
\hline & $\begin{array}{l}\text { Grund- } \\
\text { gesamtheit } \\
\text { Gymnasien }\end{array}$ & $\begin{array}{l}\text { Stichprobe } \\
\text { Gymnasien }\end{array}$ & $\begin{array}{l}\text { Grundgesamt-heit } \\
\text { Schüler \& Schülerin- } \\
\text { nen (Quelle: BfS) }\end{array}$ & $\begin{array}{l}\text { Stichprobe } \\
\text { Schüler \& } \\
\text { Schülerinnen }\end{array}$ & $\begin{array}{l}\text { Stichprobe } \\
\text { Maturaarbeiten }\end{array}$ \\
\hline $\begin{array}{l}\text { Gesamt- } \\
\text { schweiz }\end{array}$ & $100 \%(158)$ & $100 \%(30)$ & $100 \%\left(211^{\prime} 980\right)$ & $100 \%(920)$ & $100 \%(437)$ \\
\hline D-CH & $74,4 \%$ & $53,3 \%$ & $54 \%$ & $59,3 \%$ & $56.8 \%$ \\
\hline F-CH & $22,2 \%$ & $40 \%$ & $39 \%$ & $34,7 \%$ & $35.5 \%$ \\
\hline I-CH & $3,2 \%$ & $6,7 \%$ & $7 \%$ & $6 \%$ & $7.8 \%$ \\
\hline
\end{tabular}




\section{Entwicklung des Qualitätskriterienrasters}

Aufgrund fehlender systematischer Beurteilungsraster für Maturaarbeiten (Bonati \& Hadorn, 2007; Wilhelmer, 2005) wurde ein eigenes Kriterienraster zur Bestimmung der Qualität von Maturaarbeiten entwickelt. In einem ersten Entwicklungsschritt stand die Frage nach den sachlichen Bezugsnormen der Qualitätsbeurteilung im Vordergrund. Hinweise auf solche Normen liefern die Anforderungen, die von verschiedenen Seiten an Maturaarbeiten und ihre Verfasserinnen und Verfasser gestellt werden. Dies sind:

1) Anforderungen aus Sicht der gymnasialen Bildungsinstitutionen: Im Vordergrund stehen dabei vor allem die gymnasialen Bildungsziele wie sie im MAR (EDK, 1995) sowie im Rahmenlehrplan der Maturitätsschulen (EDK, 1994) beschrieben werden. Ergänzend wurde auf Erkenntnisse aus EVAMAR I (Pagnossin et al., 2005, S. 179f.) und auf Bewertungsraster einzelner Schulen zurückgegriffen.

2) Anforderungen aus Sicht der Abnehmenden (v.a. Hochschulen): Hier geht es vor allem um Fragen der Studierfähigkeit und der Hochschulreife.

3) Anforderungen aus Sicht der Disziplinen: Da eine Entwicklung verschiedener Beurteilungsraster für die einzelnen Disziplinen im Rahmen der Untersuchung nicht zu leisten war, wurden die Arbeiten durch Fachexpertinnen und -experten beurteilt, welche den Aspekt der Wissenschaftlichkeit und insbesondere der methodischen Vorgehensweise entsprechend den jeweiligen disziplinären Standards bewertet haben.

4) Anforderungen aus Sicht der Linguistik: Im Bereich der Linguistik existieren verschiedene Instrumente zur Analyse von Texten (z.B. «Zürcher Textanalyseraster", Nussbaumer \& Sieber, 1994). Auf der Basis der Überlegung, dass Texte unterschiedlichen Anforderungen zu genügen haben (Kruse, 1999), wurde das Raster nach den Anforderungsbereichen Genauigkeit, Kommunikative Funktion und Strukturiertheit aufgebaut.

Die eigentliche Entwicklung des Rasters erfolgte in einem sowohl induktiven als auch deduktiven Prozess. In seiner Endfassung besteht es aus insgesamt $32 \mathrm{Be}-$ urteilungskriterien: Neben einem Globalurteil über die ganze Arbeit, werden 31 Teilaspekte der Arbeit entlang der drei Anforderungsbereiche 1) Kommunikative Funktion, 2) Strukturiertheit und 3) Genauigkeit beurteilt. Die Operationalisierung der einzelnen Kriterien erfolgte auf einer sechsstufigen Skala, bei der jeweils die beiden Eckwerte ausformuliert wurden und der Wert 1 die beste Bewertung darstellt (vgl. Abb. 2; eine detaillierte Beschreibung des Kriterienrasters sowie der Items findet sich in Huber et al., 2008).

\begin{tabular}{|l|l|l|l|l|l|l|l|l|}
\hline Wortwahl / Wortschatz & + & & & & & - & k.A. & \\
\hline & + & 2 & 3 & 4 & 5 & 6 & 9 & $\begin{array}{l}\text { Die Wortwahl ist unsicher, } \\
\text { unbeholfen oder inhaltlich } \\
\text { nicht angemessen und we- } \\
\text { nig variabel. }\end{array}$ \\
\hline $\begin{array}{l}\text { Die Wortwahl ist } \\
\text { treffend, zielführend, } \\
\text { variabel und inhaltlich } \\
\text { angemessen. }\end{array}$ & 1 & & & & & & &
\end{tabular}

Abbildung 2: Auszug aus dem Qualitätskriterienraster 
Mittels einer explorativen Faktoranalyse mit Varimax-Rotation liessen sich aus den 31 Items drei reliable Skalen zur formalen, sprachlichen und inhaltlichen Qualität bilden (vgl. Tab. 2). Die Skala «Formale Qualität» umfasst insgesamt sieben Items (Titelblatt, Layout, Optik von Tabellen und Abbildungen, formale Gliederung, korrektes Zitieren, Quellenangaben sowie Funktionalität von Tabellen und Abbildungen) ( $\mathrm{n}=437$, Cronbach's Alpha =.75, M=2.5, SD=0.79). Die Skala sprachliche Qualität umfasst sechs Items (Textfluss, Prägnanz, Wortwahl, Ausdruck, Satzstruktur, Sprachliche Korrektheit) ( $\mathrm{n}=432$, Cronbach's Alpha=.94, M=2.5, SD=0.91) und die Skala für inhaltliche Qualität umfasst die übrigen elf Items (Titel, Feingliederung / Titelhierarchie, Umfang und Gewichtung, Schwerpunktsetzung, Kohärenz der Argumentation, Fragestellung allgemein, Überblick / Aufbau, Differenzierung, Beschreibung der Methoden, Begrifflichkeiten) ( $\mathrm{n}=437$, Cronbach's Alpha=.93, $\mathrm{M}=2.7, \mathrm{SD}=.92)$.

Tabelle 2: Korrelationsmatrix mit den drei Skalen inhaltliche, sprachliche, formale Qualität sowie dem Globalurteil der Rater

\begin{tabular}{|c|c|c|c|c|}
\hline & $\begin{array}{l}\text { Inhaltliche } \\
\text { Qualität }\end{array}$ & $\begin{array}{l}\text { Sprachliche } \\
\text { Qualität }\end{array}$ & \begin{tabular}{|l|} 
Formale \\
Qualität
\end{tabular} & Globalurteil \\
\hline Inhaltliche Qualität & & $0,67^{*}$ & $0,6^{* *}$ & $0,85^{* *}$ \\
\hline Sprachliche Qualität & $0,67^{*}$ & & $0,53^{* *}$ & $0,76^{* *}$ \\
\hline Formale Qualität & $0,6^{* *}$ & $0,53^{* *}$ & & $0,62^{* *}$ \\
\hline Globalurteil & $0,85^{* *}$ & $0,76^{* *}$ & $0,62^{* *}$ & \\
\hline
\end{tabular}

\section{Zielsetzungen zur Maturaarbeit auf nationaler und kantonaler Ebenes}

Die Analyse des Maturitätsanerkennungsreglements MAR 95 (EDK, 1995) und des Rahmenlehrplans der Maturitätsschulen (EDK, 1994) zeigte, dass hierin keine Aussagen zur konkreten Ausgestaltung der Maturaarbeit gemacht werden. Das MAR (Art. 10) definiert lediglich das «Endprodukt» (grössere schriftliche [kommentierte] Arbeit und mündliche Präsentation) und macht arbeitsorganisatorische Vorgaben: Die Arbeit kann alleine oder in einer Gruppe geschrieben werden und muss eigenständig erfolgen. Weiter gilt die Maturaarbeit als Teil des Wahlbereichs und es wird auf eine fachliche Zuordnung verzichtet (MAR, Art. 11).

Mittelbar stellen auch die in den allgemeinen Zielbestimmungen des MAR (Art. 5) sowie die im dazugehörigen Rahmenlehrplan (EDK, 1994) vermerkten Kompetenzen Richtlinien für das Verfassen von Maturaarbeiten dar - freilich ohne dass dabei direkt Bezug auf die Maturaarbeit genommen wird: Die Maturaarbeiten sollen also neben der individuellen fachlichen Vertiefung auch die Vorbereitung auf eine akademische Weiterführung der Bildungsbiographie unterstützen (EDK, 1994). 
Zusammenfassend kann festgehalten werden, dass auf nationaler Ebene konkrete und eindeutige Hinweise zu den Zielsetzungen der Maturaarbeit, zu ihrem wissenschaftspropädeutischen Bezugsrahmen sowie zu den Umsetzungsmassnahmen weitgehend fehlen. Das Fehlen einer nationalen Regelung wird auch von den Kantonen kaum kompensiert: Nur wenige der im Untersuchungssample vertretenen Kantone machen schulübergreifende Vorgaben zu Zielen, Inhalten und Abläufen bezüglich der Maturaarbeiten. Dies belässt den einzelnen Gymnasien einen grossen Gestaltungsspielraum bei der Einführung, Begleitung und Beurteilung der Maturaarbeiten, und wirft gleichzeitig Fragen der Chancengerechtigkeit auf.

\section{Rahmenbedingungen an den einzelnen Schulen und ihre Auswirkungen}

Das Fehlen übergeordneter Zielsetzungen und der damit verbundene Handlungsspielraum der Gymnasien führen in Bezug auf die Entstehungsprozesse der Maturaarbeiten zu relativ grosser Heterogenität. Diese zeigt sich einerseits im unterschiedlichen Ausmass der formalen Dokumentation, andererseits in den Inhalten von Wegleitungen, Leitfäden und Richtlinien sowie den Umsetzungsbedingungen und Bewertungsmodi.

Die Analyse der von den einzelnen Gymnasien mit einem Verbindlichkeitsanspruch formulierten und dokumentierten Informationen zeigt, dass beinahe die Hälfte aller untersuchten Schulen (43\%) in Bezug auf die Maturaarbeit eigenständige Rechtsgrundlagen in Form von Reglementen, Promotionsordnungen oder Richtlinien abfassen. Zudem benennen praktisch alle Gymnasien Ziele, die mit der Maturaarbeit erreicht werden sollen. In der Regel stehen hierbei wissenschaftliches Reflektieren und die Methodik im Vordergrund. Ausserdem wird die Maturaarbeit meist als "projektbezogene Arbeit» umschrieben, bei der die Selbstorganisation eine wichtige Rolle spielt. Interessant ist, dass die geforderte und im Zuge der MAR-Teilrevision erneut bekräftigte Interdisziplinarität bei den untersuchten Deutschschweizer Schulen - im Gegensatz zu den Schulen der Romandie - nicht explizit als Ziel formuliert wird. Ebenso betonen die Schulen der Romandie - im Gegensatz zu den Deutschschweizer Gymnasien - explizit den Aspekt des Wissenserwerbs bzw. dessen Erweiterung in ihren Zielformulierungen.

\section{Vorbereitende Veranstaltungen}

Auch in der Organisation zeigen sich zwischen den Schulen teilweise grundlegende Unterschiede. Während einige die zentralen Informationen in Informationsveranstaltungen und in Form von schriftlichen Leitfäden ausgeben, bieten andere überdies eine weite Palette von Unterstützungsangeboten (z.B. Schreibkurse, Intensivwochen, Lernen am Projekt). Die Gymnasien der lateinischen 
Schweiz organisieren die Vorbereitung und Betreuung vorwiegend in Seminarform: In eigens dafür eingerichteten Veranstaltungen wird ein meist von der Lehrperson vorgängig festgelegtes (Rahmen-) Thema behandelt, aus welchem die Schülerinnen und Schüler ihren zu bearbeitenden Gegenstand auswählen und bearbeiten.

Auf der Basis der Überlegung, dass sich vorbereitende Veranstaltungen positiv auf die Qualität der Maturaarbeiten auswirken und ausgehend von der Annahme, dass die Qualität solcher vorbereitenden Veranstaltungen zwischen den einzelnen Schulen beträchtlich divergieren können, wurden die Schülerinnen und Schüler nach dem Nutzen derartiger Veranstaltungen gefragt und geprüft, ob es einen positiven Zusammenhang zwischen der wahrgenommen Nützlichkeit der Veranstaltungen und der Maturaarbeitsqualität gibt. Die MaturandenBefragung zeigt, dass vorbereitende Veranstaltungen als nützlich empfunden werden (vgl. Abb. 3). Ebenso wurden auch die Leitfäden zum Erstellen der Arbeit von rund zwei Dritteln der Befragten als nützlich eingestuft. Selbstverständlich gibt es bei der Einschätzung zur Nützlichkeit grosse Unterschiede zwischen den Schulen, da die Schülereinschätzungen sich ja auf unterschiedliche Leitfäden beziehen (16,7\% der Varianz dabei geht auf die Schulzugehörigkeit zurück).

\section{Abbildung 3: Einschätzung der Schülerinnen und Schüler zum Nutzen vorberei-} tender Veranstaltungen

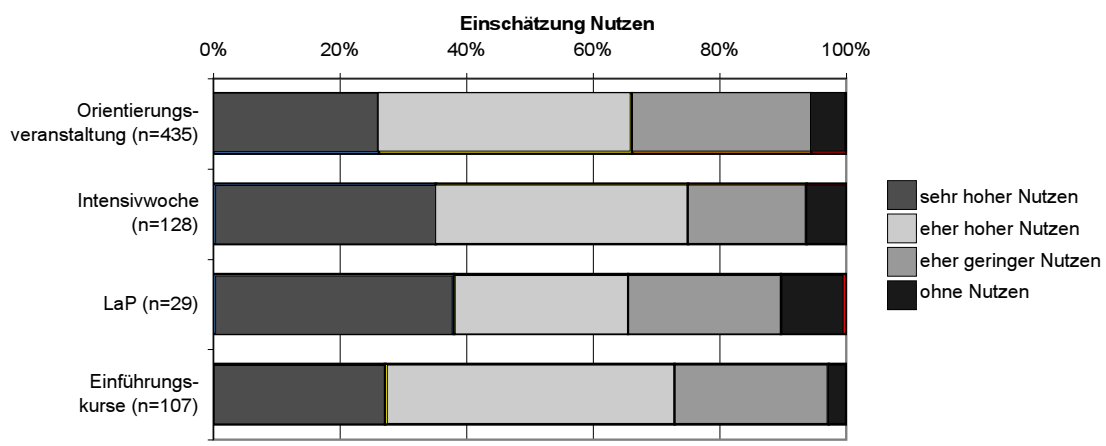

Der Nutzen von Informationsveranstaltungen wird von Maturandinnen und Maturanden mit qualitativ besseren Arbeiten positiver herausgestellt. Ausserdem fiel die durch EVAMAR II diagnostizierte inhaltliche und sprachliche Qualität von Maturaarbeiten in der Regel besser aus, wenn Schülerinnen und Schüler eine Informationsveranstaltung besucht hatten (vgl. Tab. 3). Anders die Wegleitungen oder Leitfäden zum Verfassen von Maturaarbeiten. Die Kenntnis dieser Leitfäden scheint keine Auswirkungen auf die Qualität des Endprodukts zu haben. Ein Gruppenvergleich mit t-Test zwischen der Gruppe der Maturandinnen und Maturanden, die einen Leitfaden zur Kenntnis genommen haben und de- 
nen, die diesen nicht kennen, zeigt bei keinem der Qualitätsbereiche der Maturaarbeit noch beim Globalurteil ein signifikantes Ergebnis.

Tabelle 3: Mittelwerte (Standardabweichungen) in den Qualitätsbereichen für Maturandinnen und Maturanden, die eine Infoveranstaltung besucht haben oder nicht besucht haben.

\begin{tabular}{|l|l|l|l|l|}
\hline & Formale Qualität & $\begin{array}{l}\text { Sprachliche } \\
\text { Qualität }\end{array}$ & $\begin{array}{l}\text { Inhaltliche } \\
\text { Qualität }\end{array}$ & Globalurteil \\
\hline $\begin{array}{l}\text { Infoveranstaltung } \\
\text { besucht }\end{array}$ & $2.57(.81)$ & $2.63(.95)$ & $2.86(.93)$ & $3.22(1.24)$ \\
\hline $\begin{array}{l}\text { Infoveranstaltung } \\
\text { nicht besucht }\end{array}$ & $2.50(.78)$ & $2.33(.84)$ & $2.51(.87)$ & $2.92(1.08)$ \\
\hline $\begin{array}{l}\text { Signifikanz des } \\
\text { Gruppenvergleichs }\end{array}$ & n.s. & $\mathrm{p}<0.001$ & $\mathrm{p}<0.001$ & $\mathrm{p}<0.05$ \\
\hline
\end{tabular}

\section{Themenwahl}

Die Analyse der Prozeduren zur Auswahl von Themen zeigt, dass diese an den einzelnen Gymnasien unterschiedlich gehandhabt werden. Schülerinnen und Schüler der lateinischen Schweiz etwa haben aufgrund der Organisation in Seminarform keine eigentliche Wahlfreiheit, sondern sind vielmehr durch Wahlpflicht an vorgegebene Arbeitsthemen gebunden. Anders in der Deutschschweiz, dort werden den Schülerinnen und Schülern in Bezug auf die Themenwahl weitgehende Freiheiten gelassen, was zu einer Beliebigkeit der behandelten Themen und Fragestellungen führt: Die Bandbreite umfasst denn auch quasi-wissenschaftlichen Arbeiten genauso wie Erlebnisberichte ohne wissenschaftlichen Gehalt.

Ausgehend von der Annahme, dass eine grosse Freiheit bei der Themenwahl zu höherem Interesse und damit zu einer höheren intrinsischen Motivation seitens der Studierenden führt, wurde die Hypothese formuliert, dass eine grössere Freiheit bei der Themenwahl insbesondere bei der inhaltlichen Qualität positive Auswirkungen zeigen müsse. Diese Hypothese musste jedoch aufgrund der vorliegenden Daten falsifiziert werden. Eine Varianzanalyse mit Bonferronikorrektur für Mehrfachvergleiche zeigte, dass die inhaltliche Qualität der Arbeiten bei freier Themenwahl $(M=2.8)$ signifikant $(p<0.05)$ geringer ist als bei der Vorgabe der Themen in Form einer Liste $(M=2.2)$ oder durch ein Rahmenthema $(M=2.4)$ (Huber et al., 2008). Dies ist unter Umständen darauf zurückzuführen, dass sich die Limitierung der Themen positiv auf die Zusammenarbeit zwischen den Schülerinnen und Schülern einerseits sowie zwischen den Lehrpersonen und den Schülerinnen und Schülern auswirkt. Im ersten Fall wirkt sich die grössere Nähe der jeweils zu bearbeitenden Fragestellungen positiv auf den Austausch aus. Im zweiten Fall ist davon auszugehen, dass durch die Einschränkung der Themen die fachlichen Kompetenzen der Lehrpersonen besser zum Tragen kommen. 


\section{Formale Vorgaben}

Auch im formalen Bereich sind deutliche Differenzen nach Schule erkennbar: Die Zeiträume, welche den Schülerinnen und Schülern zwischen definitiver Themenwahl und Abgabetermin für die Bearbeitung zur Verfügung stehen, variieren mit einer Dauer von 4.5 bis 15 Monaten beträchtlich, wobei der Median bei einer Dauer von 11 Monaten liegt.

Ebenso variieren die Angaben zum Umfang der Maturaarbeiten relativ stark (zwischen 10 und 30 Seiten) und es zeigte sich, dass nur wenige Schulen differenzierte Vorgaben zum Textaufbau und zur Struktur der Arbeit machen. Einigkeit herrscht einzig bezüglich der Verweise auf gängige Zitier- und Bibliographierrichtlinien.

Die Annahme eines Zusammenhangs zwischen der institutionell vorgegebenen Bearbeitungszeitspanne und der Qualität der Maturaarbeit, musste verworfen werden. Die Zeitspanne, welche für die Bearbeitung zur Verfügung steht, hat die evaluierte Qualität der Maturaarbeiten nicht signifikant beeinflusst. Ein Zusammenhang zeigte sich aber bezüglich der Zeit, welche die Schülerinnen und Schüler investiert haben: Je mehr Stunden in die Maturaarbeit investiert wurden (nachträgliche Selbsteinschätzung), desto besser wurde die Maturaarbeit bewertet. ${ }^{1}$ Kaum eine Rolle spielte dabei, ob den Maturandinnen und Maturanden Unterrichtszeit zur Bearbeitung zur Verfügung gestellt wurde (vgl. Tab. 4).

Tabelle 4: Zeitaufwand

\begin{tabular}{|l|l|l|l|}
\hline & Spearman-Rho & $\begin{array}{l}\text { Zeitaufwand für } \\
\text { Maturaarbeit }\end{array}$ & $\begin{array}{l}\text { Zeit innerhalb } \\
\text { Unterrichtsstunden }\end{array}$ \\
\hline Formale Qualität & Korrelationskoeffizient & $-.170^{* *}$ & .003 \\
\hline & Sig. (2-seitig) & .000 & .960 \\
\hline & $\mathrm{N}$ & 420 & 315 \\
\hline Sprachliche Qualität & Korrelationskoeffizient & $-.149^{* *}$ & $-.143^{*}$ \\
\hline & Sig. (2-seitig) & .002 & .012 \\
\hline & $\mathrm{N}$ & 415 & 311 \\
\hline Inhaltliche Qualtiät & Korrelationskoeffizient & $-.198^{* *}$ & -.023 \\
\hline & Sig. (2-seitig) & .000 & .689 \\
\hline & $\mathrm{N}$ & 420 & 315 \\
\hline \multirow{2}{*}{$\begin{array}{l}\text { * Die Korrelation ist auf dem Niveau von 0,01 (2-seitig) signifikant. } \\
\text { Die Korrelation ist auf dem Niveau von 0,05 (2-seitig) signifikant. }\end{array}$} \\
\cline { 2 - 2 }
\end{tabular}

\section{Betreuungssituation}

Die Betreuung geschieht in der Regel durch eine Lehrperson, wobei bemerkenswert ist, dass nur wenige Schulen die fachliche Qualifikation der betreuenden Lehrperson als conditio sine qua non explizit angeben. Es ist deshalb zu vermuten, dass neben der fachlichen Qualifikation auch die zeitliche Verfügbarkeit der Lehrpersonen sowie Fragen der Sympathie zwischen der Schülerin bzw. dem 
Schüler und der Lehrperson bei der Wahl der Betreuungsperson von Bedeutung ist. Dass die fachlichen Kompetenzen von betreuenden Lehrpersonen trotzdem zum Tragen kommen, zeigt sich in der Maturandinnen- und Maturandenbefragung: Die Mehrheit (59.1\%) der Befragten hat die Betreuungsperson aufgrund der (vermuteten oder ausgewiesenen) Kenntnis des Themas bzw. des primären Bezugsfachs gewählt.

Die Befragung hat auch gezeigt, dass von Seiten der Schülerinnen und Schüler unterschiedliche Betreuungsleistungen nachgefragt werden: Unterstützung suchten die Befragten primär in den Bereichen «Fachliche Inhalte» (53\%), «Aufbau und Gliederung der Arbeit» (51\%) sowie «Formulierung der Fragestellung» (50\%) (vgl. Tab. 5). Hierin lassen sich drei Scheitelpunkte der Beratung erkennen: Erstens die Einstiegsberatung bei der Präzisierung der Fragestellung, zweitens die Klärung inhaltlicher Fragen, die sich in einer zweiten Erarbeitungsphase stellen und drittens die Beantwortung von Fragen der Gliederung und zur Textgestaltung in einer letzten Redaktionsphase.

\section{Tabelle 5: Unterstützung durch die Lehrpersonen (Mehrfachnennungen möglich)}

\begin{tabular}{|l|l|}
\hline Bereich in dem Unterstützung gesucht wurde & Prozent der Befragten \\
\hline Inhalt der Arbeit (fachlich) & 53.3 \\
\hline Aufbau und Gliederung der Arbeit & 51.0 \\
\hline Formulierung der Fragestellung & 49.9 \\
\hline Formale Grundlagen (z.B. Fussnoten, Bibliographie, Zitieren usw.) & 37.1 \\
\hline Informationsquellen & 27.4 \\
\hline Arbeitsmethoden & 24.7 \\
\hline Zeitplanung und Arbeitsorganisation & 21.5 \\
\hline Verfassen der Arbeit (Schreibprozess) & 20.1 \\
\hline Themenwahl & 16.7 \\
\hline Motivation / Überwindung von Krisen & 12.1 \\
\hline Computer und Internetnutzung & 6.0 \\
\hline Sonstiges & 4.4 \\
\hline
\end{tabular}

In Bezug auf die Häufigkeit der Kontakte zur Betreuungsperson zeigt der Regionalvergleich, dass Tessiner Schülerinnen und Schüler mehrmals monatlich, häufig sogar mehrmals wöchentlich mit ihrer Betreuungsperson in Kontakt waren. Vermutlich ist dies auf die bereits erwähnte Seminarorganisation zurückzuführen. Etwas anders sieht die Situation in der deutsch- und französischsprachigen Schweiz aus. Hier geben jeweils mehr als die Hälfte der Schülerinnen und Schüler an, dass sie höchstens einmal im Monat mit den Lehrpersonen in Kontakt waren. Die Untersuchung zeigte entgegen den Erwartungen, dass weder die Häufigkeit der Kontakte noch die Zufriedenheit mit der Betreuungssituation in einem signifikanten Zusammenhang mit der Qualität der untersuchten Maturaarbeiten steht. Dies gilt sowohl für die formale, die sprachliche und die inhalt- 
liche Qualität als auch für das Globalurteil. Was die Frage der Häufigkeit anbelangt, können die Ergebnisse dahin gehend verstanden werden, dass es bei der Betreuung weniger um Quantität als vielmehr um die Qualität der Betreuungsleistungen geht.

\section{Bewertung}

Obwohl die Beurteilungskriterien nur in einem kleineren Teil der Schulen explizit im Leitfaden resp. den Rechtsgrundlagen genannt werden, folgt daraus nicht zwingend Unkenntnis auf Seiten der Schülerinnen und Schüler. Folgt man nämlich der Befragung von Gymnasiastinnen und Gymnasiasten zeigt sich, dass über $80 \%$ der Befragten die Beurteilungskriterien vorgängig gekannt haben. Dies lässt darauf schliessen, dass die Kriterien im Zuge der Betreuungsarbeit offen gelegt werden. Beeindruckend ist auch, dass die Schülerinnen und Schüler die Kriterien mehrheitlich (95\%) als sinnvoll einschätzten. Bedenkenswert bleibt aber der Umstand, dass auf der anderen Seite knapp 20\% der Schülerinnen und Schüler angaben, die Kriterien nicht gekannt zu haben. Rund drei Viertel dieser "Nicht-Informierten» merken zugleich an, dass sie die Kriterien gerne im Voraus gekannt hätten. Diese Befunde betonen die Bedeutung der Transparenz von Beurteilungskriterien.

Auch hinsichtlich der Gewichtung der einzelnen Arbeitsschritte und Produkte zeigen sich grosse Unterschiede zwischen den untersuchten Gymnasien: Fliesst bei 13\% der untersuchten Gymnasien die mündliche Präsentation der Maturaarbeit nicht in die Endbewertung mit ein, macht bei weiteren 13\% der Schulen die Note für die Präsentation mehr als 50\% der Gesamtnote aus. Bei den restlichen und damit der Mehrheit der Schulen (74\%) wird Präsentation weniger stark gewichtet. Die Mehrheit der Schulen (63\%) verweist zudem darauf, dass auch der Arbeitsprozess in die Beurteilung mit einbezogen wird.

Die 2007 erfolgte Teilrevision des MAR legt nun fest, dass in jedem Fall die schriftliche Arbeit, die mündliche Präsentation und der Arbeitsprozess zur Bewertung hinzugezogen werden müssen. Letzterer wird gegenwärtig in allen untersuchten Schulen anhand eines «Arbeitsjournals» dokumentiert, das als Begleitinstrument genutzt wird. Inwiefern die Teile Dokumentation, Präsentation und Arbeitsprozess jedoch gewichtet werden, bleibt auch nach der Teilrevision den Schulen überlassen. Angesichts der massiven Unterschiede in der Praxis, stellt sich hier die Frage, ob nicht einheitliche Vorgaben hinsichtlich der Gewichtung gemacht werden sollten. Derartige Vorgaben setzen aber die Klärung der Zielsetzung, die mit der Maturaarbeit verbunden ist, voraus. Denn steht der wissenschaftspropädeutische Charakter im Vordergrund, müsste die schriftliche Arbeit stärker gewichtet werden. Soll jedoch mit der Maturaarbeit gezeigt werden, dass man selbständig ein Projekt durchführen kann, dann müsste wohl das Element Arbeitsprozess eine stärkere Gewichtung erfahren. Eine andere Frage, die sich im Zusammenhang mit der Bewertung stellt, ist die Vermischung von formativer und summativer Evaluation. 
Ein Blick auf die Bewertungspraxis zeigt, dass nur 1.3\% der untersuchten Maturaarbeiten von den Lehrpersonen als ungenügend eingestuft wurden. Fast die Hälfte (47\%) aller Arbeiten wurde - gemäss Aussagen der Maturandinnen und Maturanden - mit dem Prädikat «sehr gut» bewertet. Die Untersuchung der Qualität von Maturaarbeiten durch die Raterinnen und Rater von EVAMAR II ergab ein kritischeres Bild. Diese Unterschiede erklären sich teilweise aus dem Untersuchungsdesign: So wurde im Rahmen von EVAMAR II nur die schriftliche Arbeit untersucht - weder der Arbeitsprozess noch die mündliche Präsentation konnten mitberücksichtigt werden. Deutlich wird aber auch, dass die konsequent wissenschaftspropädeutische Ausrichtung der Beurteilung, wie sie in EVAMAR II eingesetzt wurde, nicht oder nur teilweise als Leitfaden bei der Schlussbeurteilung durch die Lehrpersonen gilt. Schliesslich zeigt sich in der Differenz der Beurteilung aber auch ein anderes, ebenfalls bekanntes Problem: Lehrpersonen beurteilen bei der Maturaarbeit nicht nur diese, sondern bewerten ein Stück weit immer auch ihre eigene Betreuungspraxis mit, was zu Gefälligkeitsnoten führen kann. Ausserdem bildet sich im Laufe der Betreuungsarbeit eine Art «anwaltschaftliche Parteinahme heraus», d.h. «der Betreuer oder die Betreuerin ist bereit, den betreuten Schüler gegen Kritik von Aussen (zum Beispiel der zweitbeurteilenden Person) zu verteidigen» (Hadorn, 2008, S. 2).

\section{Fazit: Notwendige Klärung der Zielsetzung}

Die Maturaarbeit zählt seit der Teilrevision 2007 des MAR zur Bestehensnorm der gymnasialen Matura. Einleitend haben wir die Frage nach der Vergleichbarkeit von Maturaarbeiten und ihren Bewertungen aufgeworfen. Bis anhin wurde und wird - aufgrund fehlender übergreifender Ziel- und Vollzugsvorgaben - die Beurteilung der Maturaarbeit an jeder Schule, bisweilen auch von jeder Betreuungsperson unterschiedlich gehandhabt. Die auf interkantonaler Ebene kaum und auf einzelschulischer Ebene sehr unterschiedlich formulierten Zielsetzungen für die Maturaarbeiten verweisen auf die unterschiedliche Auslegung der Frage nach möglichen Anforderungen und erwünschten Leistungen, welche durch die Schülerinnen und Schüler mit der Maturaarbeit erbracht werden sollen. Gleichzeitig erscheint es unbestritten, dass mit der Maturaarbeit ein wissenschaftspropädeutischer Beitrag geleistet werden soll, denn das Einüben von «wissenschaftlichem Arbeiten» findet sich als allgemeines Ziel in beinahe allen Dokumentationen der untersuchen Gymnasien. Eine einheitliche Auslegung der Konzepte Wissenschaftspropädeutik und Studierfähigkeit sowie die Formulierung einheitlicher Beurteilungskriterien stehen aber aus.

Angesichts dieser Ausgangslage verblüfft es kaum, dass sich die Benotungsbasis höchst unterschiedlich präsentiert. Erstaunlich scheint aber, dass die Beurteilungspraxis für die Maturaarbeit im Fachdiskurs bisher wenig Aufmerksamkeit erfährt. Zwar wurde bei der 2007 erfolgten Teilrevision des MAR festgelegt, dass 
neben der schriftliche Arbeit, auch die mündliche Präsentation und der Arbeitsprozess zur Bewertung hinzugezogen werden müssen. Das heisst, eine Vermischung formativer und summativer Bewertung wird - zumindest bildungspolitisch - explizit gewünscht. Ob dies aber auch sinnvoll ist, kann letztlich nur beurteilt werden, wenn die Zielsetzungen, die mit der Maturaarbeit verbunden sind, geklärt sind. Sofern auf die Vermischung formativer und summativer Evaluation nicht verzichtet werden soll, müsste zudem - alleine schon aus Chancengleichheitsgründen - die Gewichtung der drei Teilaspekte verbindlich festgelegt werden.

Eine Klärung der Zielsetzung ist jedoch nicht nur im Hinblick auf die Beurteilungspraxis angezeigt, sondern auch im Hinblick auf die Organisation der Maturaarbeit an den einzelnen Schulen. Steht etwa die wissenschaftspropädeutische Bedeutung der Maturaarbeit im Vordergrund, müsste - vor dem Hintergrund der oben dargestellten Erkenntnisse - über eine allfällige Einschränkung der Themenwahl (Wahlpflicht) und die Einführung des «Seminarmodells» nachgedacht werden. Soll die Maturaarbeit aber eher dem Erwerb von Projektmanagementkompetenzen oder dergleichen dienen, dann ist eine grössere Freiheit bei der Themenwahl durchaus angemessen und eine allenfalls geringere Qualität bei den schriftlichen Endprodukt durchaus verkraftbar.

\section{Anmerkungen}

1 Diesen Zusammenhang hat eine Neuberechnung der Daten ergeben. Die Angaben die im Abschlussbericht von EVAMAR II (vgl. Huber et al., 2008, S. 348) publiziert wurden, müssen entsprechend berichtigt werden.

\section{Literatur:}

Binder, H.-M. \& Feller-Länzlinger, R. (2003). Maturaarbeit und Maturaprüfung nach neuem Maturitätsanerkennungsreglement MAR. Auswertung der schriftlichen Befragung der AbsolventInnen der Matura 2002 im Rahmen der externen Evaluation der Gymnasialreform im Kanton Luzern. Luzern: Interface, Institut für Politikstudien.

Binder, H.-M. \& Feller-Länzlinger, R. (2004). Maturitätsreform im Kanton Aargau. Schlussbericht der externen Evaluation. Luzern: Interface, Institut für Politikstudien.

Bonati, P. \& Hadorn, R. (2007). Matura- und andere selbständige Arbeiten betreuen. Ein Handbuch für Lehrpersonen und Dozierende. Bern: h.e.p.

Davaud, C. \& Hexel, D. (2003). La nouvelle maturité gymnasiale. Orientations et intérêts des élèves de 4e. Genève: Service de la Recherche en Education. Consulté le 19 décembre 2007 dans http://www.ge.ch/recherche-education/doc/publications/docsred/2003/nouv-matugym.pdf

Dreyer, H. P. (2007). VSG. Jahresbericht 2006/2007. Gymnasium Helveticum, 2006 (3), 16-21.

Eberle, F., Gehrer, K., Jaggi, B., Kottonau, J., Oepke, M., Pflüger, M., Huber, C., Husfeldt, V., Lehmann, L. \& Quesel, C. (2008). Evaluation der Maturitätsreform 1995 (EVAMAR). Schlussbericht zur Phase II. Bern: Staatssekretariat für Bildung und Forschung.

EDK. (Hrsg.). (1972). Mittelschule von Morgen. Jahrbuch der Schweizerischen Konferenz der kantonalen Erziehungsdirektoren, 58.

EDK. (1994). Rahmenlehrplan für die Maturitätsschulen vom 9. Juni 1994. Empfehlungen an die Kantone gemäss Art. 3 des Schulkonkordats vom 29. Oktober 1970. Mit Handreichung zur Umsetzung. Bern: EDK. 
EDK. (1995). Verordnung des Bundesrates/Reglement der EDK über die Anerkennung von gymnasialen Maturitätsausweisen (MAR) vom 16. Januar/15. Februar 1995.

EDK. (2007). Stärkung der Naturwissenschaften und Aufwertung der Maturaarbeit in der gymnasialen Ausbildung. Medienmitteilung vom 27. Juni 2007. Bern: EDK.

Hadorn, R. (2008). Unterlagen zu Referat 1: Betreuung und Bewertung von MaturaarbeitenRollenkonflikt oder ganzheitliche Aufgabe? und zu Atelier 3: Bewertungskriterien entwickeln, vereinbaren und anwenden. Biel: WBZ. Zugriff am 04.05.2009 unter http://www.tmma.ch/Archiv/Referat_Hadorn_d.pdf

Heldmann, W. (1998). Studieren heute. Erwartungen der einzelnen Studienfächer an ihre Studienanfänger (2., völlig neubearb. Aufl.). Bad Honnef: Bock.

Huber, C., Husfeldt, V., Lehmann, L. \& Quesel, C. (2008). Teilprojekt D2: Die Qualität von Maturaarbeiten in der Schweiz. In F. Eberle et al., Evaluation der Maturitätsreform 1995 (EVAMAR). Schlussbericht zur Phase II (S. 277-365). Bern: Staatssekretariat für Bildung und Forschung.

Huber, L. (1997). Fähigkeit zum Studieren - Bildung durch Wissenschaft? Zum Problem der Passung zwischen Gymnasialer Oberstufe und Hochschule. In E. Liebau, W. Mack \& Ch. Scheilke (Hrsg.), Das Gymnasium. Alltag, Reform, Geschichte, Theorie (S. 333-351). München: Juventa.

KMK [Ständige Konferenz der Kultusminister der Länder in der Bundesrepublik Deutschland]. (2006). Vereinbarung zur Gestaltung der gymnasialen Oberstufe in der Sekundarstufe II. Beschluss der Kultusministerkonferenz vom 07.07.1972 i.d.F. vom 02.06.2006. Letzter Zugriff am 27.02.2008 unter http://www.kmk.org/fileadmin/veroeffentlichungen_beschluesse/1972/1972_07_07-Vereinbarung-Gestaltung-Sek2.pdf

Konegen-Grenier, C. (2002). Studierfähigkeit und Hochschulzugang. Köln: Deutscher Instituts-Verlag.

Kruse, O. (1999). Keine Angst vor dem leeren Blatt. Ohne Schreibblockade durchs Studium (7. Auflage). Frankfurt: Campus.

National Academy of Science (1996). National science education standards. Retrieved February 07, 2008 from http://www.nap.edu/readingroom/books/nses/2.html\#dsl

Nussbaumer, M. \& Sieber, P. (1994). Texte analysieren mit dem Zürcher Textanalyseraster. In P. Sieber (Hrsg.), Sprachfähigkeiten - besser als ihr Ruf und nötiger denn je! Ergebnisse und Folgerungen aus einem Forschungsprojekt (S. 142-185). Salzburg: Sauerländer.

Pagnossin, E., Alliata, R. \& Dozio, E. (2005). Le travail de maturité. In E. Ramseier, J. Allraum \& U. Stalder, Evaluation der Maturitätsreform 1995 (EVAMAR). Neue Fächerstruktur, Pädagogische Ziele, Schulentwicklung. Schlussbericht zur Phase 1 (S. 171-191). Bern: Staatssekretariat für Bildung und Forschung.

Schmidt, A. (1994). Das Gymnasium im Aufwind. Entwicklung, Struktur, Probleme seiner Oberstufe. Aachen-Hahn: Hahner.

Trautwein, U. \& Lüdtke, O. (2004). Aspekte von Wissenschaftspropädeutik und Studierfähigkeit. In O. Köller, R. Watermann, U. Trautwein \& O. Lüdtke (Hrsg.), Wege zur Hochschulreife in Baden-Württemberg. TOSCA - Eine Untersuchung an allgemein bildenden und beruflichen Gymnasien (S. 327-366). Opladen: Leske und Budrich.

USO [Union der Schülerorganisationen]. (2006). Schülerorganisationen: Endlich, zählt' die Maturaarbeit! Medienmitteilung der USO vom 27.12.2006. Bern: USO.

Wilhelmer, H. (2005). Fach(bereichs)arbeiten an berufsbildenden Schulen. Besichtigung eines Modells am Beispiel der HLW und Modeschule Klagenfurt. Informationen zur Deutschdidaktik (ide), 3, 84-88.

Wolter, A. (1997). Das deutsche Gymnasium zwischen Quantität und Qualität. Die Entwicklung des Gymnasiums und der Wandel gesellschaftlichen Wissens. Bibliotheks- und Informationssystem der Universität Oldenburg. 
Schlagworte : Gymnasiale Maturität, Maturaarbeit, Evaluation

\section{Les travaux de maturité gymnasiale: de différentes conditions de réalisation}

\section{Résumé}

Le règlement de reconnaissance de la maturité de 1995, conçu par la confédération et les cantons, a été partiellement réformé en 2007. Cette réforme partielle a notamment concerné le statut du travail de maturité introduit dans le règlement de 1995. Alors que ce travail était intégré dans la formation précédant les examens de maturité, il compte désormais aussi dans la note finale de la maturité gymnasiale. Étant donné ce changement de statut, il est donc nécessaire d'examiner la comparabilité des travaux de maturité et de leur évaluation. Dans le cadre de l'étude d'évaluation de la réforme de la maturité, la formation gymnasiale a été évaluée pour la seconde fois dans toute la Suisse. Or, les travaux de maturité ont cette fois-ci aussi fait l'objet d'un examen approfondi. L'article présente certains résultats issus de cette étude d'évaluation.

Mots-clés: Maturité gymnasiale, travail de maturité, évaluations

\section{Diverse condizioni per la redazione del lavoro di maturità}

\section{Riassunto}

La regolamentazione del riconoscimento del diploma di maturità, definite nel 1995 dalla Confederazione e dai Cantoni, è stata in parte modificata nel 2007. Questa parziale riforma ha riguardato, tra gli altri, il lavoro di maturità già introdotto nella prima regolamentazione del 1995. Mentre prima il lavoro di maturità era parte del programma prima degli esami di maturità, viene ora considerato come parte della valutazione finale di maturità. Per questo, diventa importante esaminare quanto il lavoro di maturità e le modalità di valutazione siano comparabili tra i diversi cantoni. Nel contesto della valutazione della riforma, il liceo è stato valutato una seconda volta in tutta la Svizzera. Gli esami di maturità sono stati esaminati in profondità. Questo articolo presenta alcuni risultati selezionati dal più ampio progetto di valutazione.

Parole chiave: Liceo, lavoro di maturità, valutazione 


\section{Variable conditions in the implementation of the baccalaureate essay}

\section{Abstract}

The 1995 regulations of baccalaureate diploma acknowledgment, which are set by the Swiss confederation together with the cantons, have partly been reformed in 2007. This partial reform concerned, among others, the baccalaureate essay first introduced in the 1995 regulations. While it only used to be part of the education program preceding the maturity exams, it now also counts into the final maturity grade. Therefore, it is necessary to examine to what extent the baccalaureate essays and the way they are assessed are comparable over cantons. Within the context of the baccalaureate reform evaluation procedure, baccalaureate education has been evaluated all over Switzerland for the second time. The baccalaureate essays have been examined in depth. This contribution presents some selected results the assessment study yielded.

Key words: Baccalaureate education, baccalaureate essay, evaluation 